IP Periodica Polytechnica Chemical Engineering

60(4), pp. 218-230, 2016

DOI: $10.3311 /$ PPch.9470

Creative Commons Attribution (i)

RESEARCH ARTICLE

\section{Analytical Approaches for the Quantitation of Redox-active Pyridine Dinucleotides in Biological Matrices}

\author{
Anna Somogyi ${ }^{1}$, George Horvai ${ }^{1,2}$, Miklós Csala $^{3}$, Blanka Tóth $^{1 *}$
}

Received 12 May 2016; accepted after revision 24 May 2016

\begin{abstract}
Some of the main electron carriers in the metabolism are mono- or dinucleotides and they play crucial roles in maintaining a balanced redox homeostasis of cells, and in coupling many anabolic and catabolic reactions. Altered cellular redox status can be an indicator of various metabolic disorders such as obesity, the metabolic syndrome, or type 2 diabetes and of other pathological conditions, which involve oxidative stress, such as cardiovascular diseases. Adequate $N A D^{+} / N A D H$ and $N A D P^{+} / N A D P H$ ratios are fundamental for normal cellular functions, thus accurate measurement of these pyridine dinucleotides is essential in biochemical research. Liquid chromatography coupled to tandem mass spectrometry has become the leading analytical technology in (targeted) state-of-the-art metabolic profiling. Main difficulties that hamper quantification of metabolites are chemical similarities, high polarity, and chemical and biological instability of the molecules to be measured. In this review, some critical steps of studying cellular redox status are described, in particular, different techniques of sample preparation and challenges in chromatographic separation.
\end{abstract}

\section{Keywords}

nicotinamide adenine dinucleotide, redox couple, separation, HPLC, mass spectrometry, method

\footnotetext{
${ }^{1}$ Department of Inorganic and Analytical Chemistry, Budapest University of Technology and Economics, H-1111 Budapest, Hungary

${ }^{2}$ MTA-BME Research Group of Technical and Analytical Chemistry, H-1111 Budapest, Hungary

${ }^{3}$ Department of Medical Chemistry, Molecular Biology and

Pathobiochemistry, Semmelweis University, H-1144 Budapest, Hungary

${ }^{*}$ Corresponding author, e-mail: tblanka@mail.bme.hu
}

\section{Introduction}

In the last few decades, more and more attention has been paid to the role of redox active nucleotides in the metabolism. Their enormous importance in the cellular antioxidant defense is undebated; flavin and nicotinamide nucleotides are essential in energy transduction as main electron transfer molecules and substrates for over 700 oxidoreductase enzymes [1]. Severe disturbance in the redox balance, e.g. oxidative stress, which is often caused by an external impact is an important component of the pathomechanism of cardiovascular diseases such as heart failure, myocardial ischemia, unstable angina and ischemia reperfusion injury [2]. The largely autonomous redox homeostasis of the endoplasmic reticulum (ER) is based on the co-localization of a reduced pyridine dinucleotide $\mathrm{NAD}(\mathrm{P})^{+} / \mathrm{NAD}(\mathrm{P}) \mathrm{H}$ system and an oxidized thiol-disulfide system. The former maintains a local cortisol production in many glucocorticoid target cells (e.g. hepatocytes, muscle cells and adipocytes), while the latter is indispensable for generation of intrachain and interchain protein disulfide bridges and hence for the appropriate protein folding in the ER lumen. When the ER redox conditions are affected by cellular disturbance, inadequacy of the protein processing machinery leads to the luminal accumulation of immature proteins. This ER stress stimulates a complex signalling network referred to as the unfolded protein response (UPR), which is primarily adaptive, but it can also lead to different destructive downstream effects including inflammations, apoptosis and insulin resistance [3]. Ratio of oxidized and reduced forms of pyridine dinucleotides gives precious information about redox metabolism disorders or energetic alterations [2]. Thus, precise measurement of these nucleotides has become essential.

Since the development of liquid chromatography (LC), its increasing penetration in biological and clinical analysis is obvious. LC coupled to UV detection was a common tool in analytics, but its application is limited by its relatively low sensitivity and specificity. Development of mass spectrometry (MS) and its coupling to LC seems to solve the majority of these problems. MS is up to several orders of magnitudes more sensitive than UV detection, and it simplifies separation as well, as it can differentiate compounds of equal retention time. However, MS needs changes 
in separation techniques since use of non-volatile salts such as phosphates, is not advisable in MS. The most suitable mobile phases usually consist of methanol or acetonitrile and water with a buffer of volatile salts (i.e. ammonium-acetate, ammonium formate) and in some cases, volatile ion-pair reagents, among others dibutylamine (DBA) [4], tributylamine (TBA) [5-7], dimethylhexylamine (DMHA) [8]. High resolution is fundamental in untargeted metabolomics, but in targeted metabolic profiling, lower resolution tandem mass spectrometers, such as triple quadrupoles also provide the information needed [9].

The novel detection mode requires new or modified techniques to be considered for sample pre-treatment as well. Although a generic method consisting of protein precipitation and extraction may sometimes be sufficient, the efficiency of the analysis can be largely improved by optimization of sample preparation for the selected metabolites.

The purpose of this review is to collect and critically compare the most common practices in profiling cellular metabolic and redox status using LC coupled to tandem MS, focusing particularly on sample pre-treatment and different separation techniques such as using an ion-pair reagent, or HILIC-HPLC.

\section{Metabolism and roles of pyridine nucleotides}

Pyridine dinucleotide coenzymes, such as $\mathrm{NAD}^{+}$and $\mathrm{NADP}^{+}$, play crucial roles in intracellular metabolism as they serve as electron carriers for several enzymes. External impacts, for instance overfeeding, starving, alcohol ingestion or drug treatments usually affect the intracellular redox state of these coenzymes and therefore monitoring the levels of their oxidized and reduced forms (i.e. $\mathrm{NAD}(\mathrm{P})^{+}$and $\mathrm{NAD}(\mathrm{P}) \mathrm{H}$, respectively) (Fig. 1) can provide information on the effect of dietary factors or drug candidates [2].

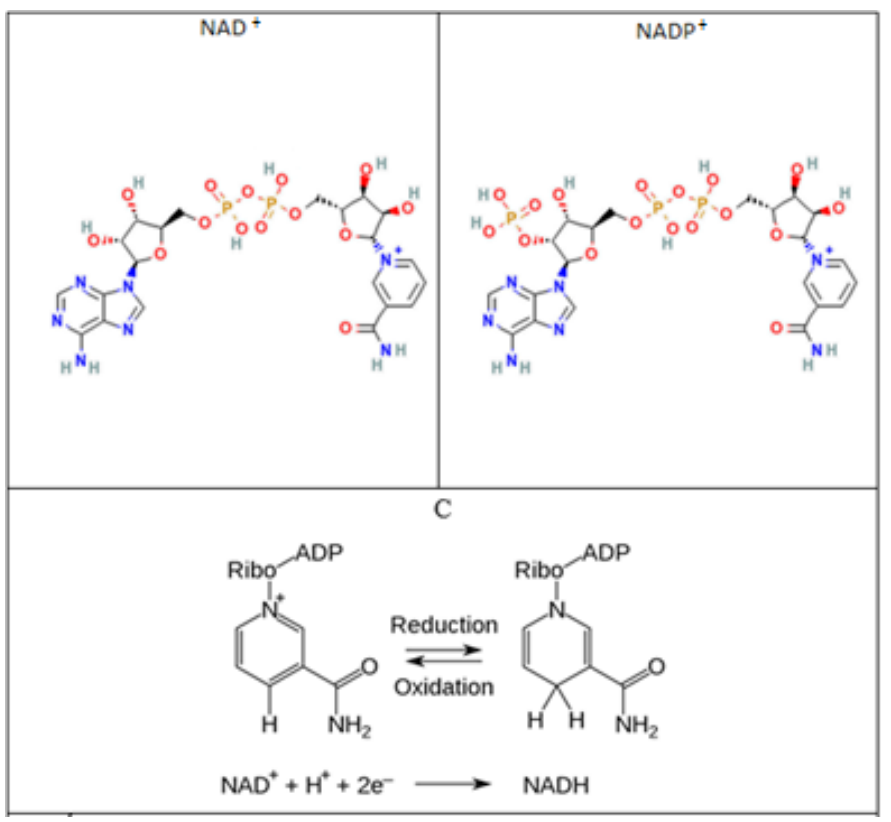

Fig. 1 Chemical structure of $\mathrm{NAD}^{+}$and $\mathrm{NADP}^{+}$, and their nicotinamide group carrying the hydride ion.
One of the most important coenzymes in hydride transfer reactions is nicotinamide adenine dinucleotide $\left(\mathrm{NAD}^{+}\right)$. It is a substrate of most dehydrogenase enzymes involved in nutrient catabolism, and its reduced form, NADH delivers the collected electrons preferentially to mitochondrial oxidative phosphorylation. As $\mathrm{NAD}^{+}$is essential in fuel utilization, protein modification and in cell signalling, information on its concentration and redox state in different cells and tissues can help understand biochemical functions, metabolic state of cells and effects of drugs in the organism [9].

Cytoplasmic $\mathrm{NAD}^{+} / \mathrm{NADH}$ ratio normally lies around 1100 [10] in aerobic cell; and it shows that $\mathrm{NAD}^{+}$usually functions as an oxidative agent in biochemical processes such as fatty acid oxidation, glycolysis, and citrate cycle. Thus, change in $\mathrm{NAD}^{+} / \mathrm{NADH}$ ratio can indicate changes in metabolic processes and in several diseases [1]. In 2013, Gomes et al. proved that $\mathrm{NAD}^{+}$plays an essential role in anti-aging processes, and elevation of $\mathrm{NAD}^{+}$levels in old mice restores mitochondrial function to that of a young mouse in a SIRT1-dependent manner [11]. Therefore regular monitoring of $\mathrm{NAD}^{+}$levels can improve our knowledge about the mechanism of age-associated diseases [12].

\section{Endoplasmic reticulum and its redox environment}

The ER is a metabolic compartment which participates in many fundamental pathways of the intermediary metabolism [3]. The organelle is a continuous membrane network separating a narrow lumen from the cytosol. A large number of enzymes are embedded in the ER membrane, and many of them expose their active sites on the cytosolic surface. However, the ER lumen also gives place to the active centres of several membrane-bound or particulate enzymes, including many oxidoreductases [13]. The concentration and redox state (i.e. the ratio of oxidized and reduced forms) of the redox couples can be remarkably different between the cytosolic and luminal environment [14]. Although the $\mathrm{NADP}^{+}-\mathrm{NADPH}$ couple is dominantly reduced in both compartments, the maintenance of this reduced state is based on different and independent enzyme activities. Moreover, the thiol-disulfide couple is actively oxidized in the ER lumen, and consequently the disulfide/thiol ratio is much higher in the ER than in the cytosol. In case of varying metabolic conditions, cells can modify ER functions. Extreme conditions such as over- or undernutrition, hypoxia or lipotoxicity [15] can cause redox imbalance and ER stress, which contributes to apoptosis, inflammation and insulin resistance via activation of the UPR. The main reason of obesity, metabolic syndrome and type 2 diabetes is the stimulation of local activation of glucocorticoids $[3,16]$. Cytoplasmic $\mathrm{NADP}^{+} / \mathrm{NADPH}$ ratio was shown to be around 0.01 [10]; in accordance with the fundamental role of this redox couple as a readily available reducing source for biosynthesis, biotransformation and antioxidant defense. It is widely accepted that the major redox buffer of the ER is composed 
of pyridine dinucleotides, especially $\mathrm{NADP}^{+}$and its reduced form, NADPH, but presence of flavin nucleotides, ascorbate and other redox-agents was shown too [17]. The cytosolic $\mathrm{NADP}^{+}$-NADPH pool is practically separated from that of the ER because the ER membrane has a limited and selective permeability, and the transmembrane traffic of pyridine dinucleotides is very slow compared to their metabolic interconversion [18]. In other words, the ER luminal reductases are uncoupled from the cytosolic NADPH generating mechanisms, and they cooperate with local $\mathrm{NADP}^{+}$-dependent dehydrogenases. It is due to the membrane impermeability that the microsomes, i.e. the artificial vesicles produced from the ER upon cell or tissue fractioning, stably retain the luminal pyridine dinucleotides and still possess the luminal redox couplings.

Measurement of all the linked redox components and determination of a redox environment in its entirety is not possible, hence changes of a complex redox system are studied by monitoring a representative redox couple as an indicator [14]. Measurement of the microsomal (ER luminal) pyridine dinucleotides would be greatly informative regarding the local redox conditions. In vivo or cellular monitoring of $\mathrm{NAD}^{+} / \mathrm{NADH}$ and $\mathrm{NADP}^{+} / \mathrm{NADPH}$ cannot be performed yet, therefore all available data have been collected from isolated microsomes. However, the precise and reliable quantitative analysis of microsomal pyridine dinucleotides has not been solved yet either, hence the intravesicular pyridine dinucleotide redox state is usually monitored in an indirect manner by using enzymatic methods [17]. These methods take advantage of the presence of intraluminal oxidoreductases which use pyridine nucleotides as co-substrates. Decades ago, pyridine nucleotide content of ER-derived microsomal vesicles was proved by Bublitz et al. [19] Odermatt et al. [20] performed a study in which functions of the ER enzyme $11 \beta$ hydroxysteroid

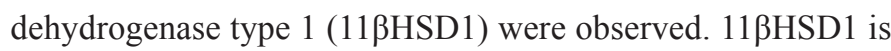
responsible for the interconversion of cortisone and cortisol in the lumen of ER (Fig. 2). Although in vitro this enzyme can act both in oxidative and reductive reaction, it was shown that in vivo, this reversible reaction is shifted towards cortisone reduction [18]. This observation suggests the dominance of NADPH in the lumen, and hexose-6-phosphate dehydrogenase (H6PD) can help maintain this ratio [14]. Lavery et al. found that in H6PD knockout mice, 11ßHSD1 mediated glucocorticoid generation was not performed [13], hence separate luminal pyridine nucleotide pool must exist to satisfy NADPH need of the reduction [14]. Alterations in the ER luminal pyridine dinucleotide redox state are usually deduced from the changes in the intrinsic cortisone reducing and cortisol oxidizing capacity of the microsomes. The results of such measurements are usually in accord with the modifications of endogenous $\operatorname{NAD}(\mathrm{P})(\mathrm{H})$ fluorescence of the intact microsomal vesicles; nevertheless, these approaches do not yield data of appropriate accuracy and reproducibility, and a significant improvement can be expected from direct measurements of individual redox components $\left(\mathrm{NAD}^{+}, \mathrm{NADP}^{+}, \mathrm{NADH}\right.$ and NADPH$)$.

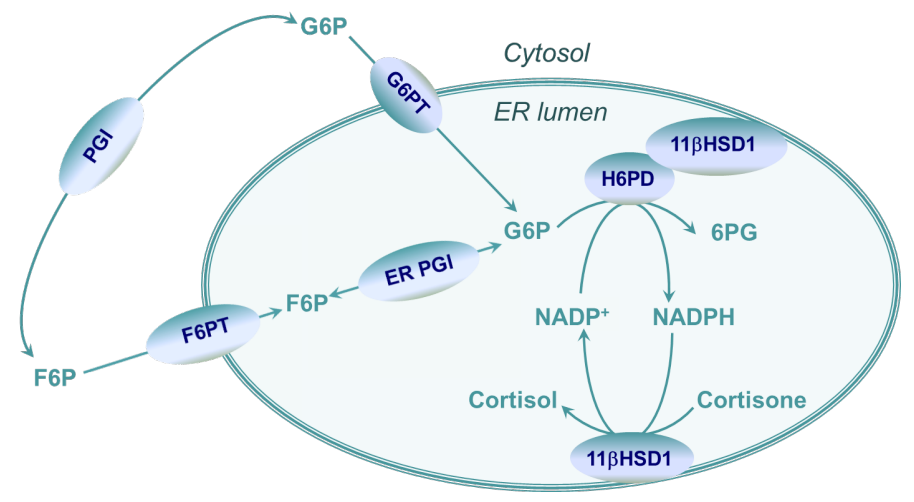

Fig. 2 Pyridine dinucleotide homeostasis in the ER lumen. F6P, fructose 6-phosphate; F6PT, F6P transporter; G6P, glucose 6-phosphate; G6PT, G6P transporter; H6PD, hexose 6-phosphate dehydrogenase; $11 \beta \mathrm{HSD} 1,11 \beta$ hydroxysteroid dehydrogenase type 1; 6PG, 6-phosphogluconate; PGI, phosphoglucose isomerase.

\section{Analysis of endogenous pyridine dinucleotides}

As mentioned above, endogenous concentration of pyridine dinucleotides can vary within wide ranges. Numerous methods were studied for measurement of pyridine and adenine nucleotides, in which the leading methods were HPLC methods coupled to different detecting systems, such as HPLC-UV [2, 21-24] HPLC-NMR, or enzymatic assays. Beside the most common methods, other type of measurement of nucleotides were suggested, such as capillary zone electrophoresis [25, 26], or spectrophotometric method for the determination of intracellular NAD(P)H [27]. But recently, LC coupled to mass spectrometry (LC-MS) has become the most important analytical method in metabolic profiling and quantitation of these small molecules in complex matrices. Untargeted metabolomics need high mass resolution instruments such as mass spectrometers with time of flight or ion-cyclotron resonance detecting systems, in targeted quantitative LC-MS, use of triple quadrupole (QQQ) as a lower resolution tandem mass spectrometers can satisfy the requirements. QQQ systems can provide multidimensional data ( $\mathrm{m} / \mathrm{z}, \mathrm{MS} 2$ transitions in MRM mode and retention time), thus metabolites which differ only in one hydrogen atom can also be distinguished. With the development of stationary phases and separation columns and the use of sub-2- $\mu \mathrm{m}$ particles, chromatographic resolution has been enhanced [28].

Most of the published studies aimed a multiple metabolite profiling, which involves the examination of many different metabolite groups. Several groups assess the phosphoryl metabolites, such as adenine nucleotides, but pyridine dinucleotides, $\mathrm{NAD}(\mathrm{H})$ and $\mathrm{NADP}(\mathrm{H})$ rarely constitute the main subject of these studies. Hence, in many cases, sample pre-treatment and LC are not optimised directly to these compounds. This review focuses on the methods for the determination of often neglected pyridine dinucleotides. 


\section{Sample pre-treatment}

Nucleotide profile has been investigated in various matrices in recent years. Metabolism of Escherichia coli (E.coli) [5, 6, 29-31], Saccharomyces cerevisiae (Sacch. cer.) [9, 31, 32], and Methylobacterium extorquens AM1 [33] was monitored, and whole blood [2, 12], serum and plasma [6, 7, 34] urine [6], cultured cells $[6-9,30,34,35]$, cerebrospinal fluid [34], and tissues $[4,6,7,31,34]$ were used as samples.

The measurement usually starts with the quick quenching of metabolism. In living cells, concentration and ratio of the monitored molecules can change within seconds, so keeping the original state of cells is fundamental in profiling. Several methods are described to stop metabolism, such as rapid cooling, especially quick freezing in liquid nitrogen for tissues, and mixing the cultured cells with organic solvents [29] (in many cases with cold organic solvent) [5] are often used. Less common methods, but still with importance, are the heat treatment of the system [31,32], and acidification. But while considering the use of these techniques, it must be ensured that all the analytes to be examined are heat or acid stable. After quenching the metabolism, the extraction procedure is the critical step. A wide variety of extraction methods can be found; the nature of the metabolites to be determined and the sample type have the biggest impact in choosing the most appropriate protocol. When the analytes have been extracted, samples are usually kept at $-20{ }^{\circ} \mathrm{C}$ or $-80^{\circ} \mathrm{C}$.

A generally accepted extraction method for Escherichia coli usually has a quenching step with the use of 80:20 methanol: water [29, 30], ethanol [5], methanol: acetonitrile: water [6]. After several centrifugation - resuspension - ultrasound cycles, in which the supernatant is always removed and stored, the final extract is then stored at $-20^{\circ} \mathrm{C}$ or $-80^{\circ} \mathrm{C}$. In one case, $0.3 \mathrm{M} \mathrm{KOH}$ was added to the extract which was later neutralized with glacial acetic acid [5]. For whole broth extraction, aqueous $75 \%(\mathrm{v} / \mathrm{v})$ ethanol solution preheated to $78{ }^{\circ} \mathrm{C}$ was mixed with E.coli culture, and was incubated at $78^{\circ} \mathrm{C}$ for $1 \mathrm{~min}$, then frozen in liquid nitrogen. After thawing and centrifugation, the supernatant was dried and stored at $-80^{\circ} \mathrm{C}$ until re-suspension [31].

Extraction methods for Saccharomyces cerevisiae are usually very similar to methods for E.coli. Preheated at $95^{\circ} \mathrm{C}, 75 \%$ ethanol was used for extraction followed by drying - resuspension - centrifugation to obtain nucleotides from yeast culture [31]. In another case, precooled, aqueous $60 \%(\mathrm{v} / \mathrm{v})$ methanol buffered with $10 \mathrm{mM}$ ammonium acetate $(\mathrm{pH} 7.5)$ was used as an extraction solvent [32].

Heat treatment was applied for extraction of nucleotides from Methylobacterium extorquens AM1 [33]. After centrifugation of quenched biomass, boiling HEPES buffered ethanol: water 75:25 at pH 5.2 was mixed with cell pellet. After two centrifugation cycles, supernatant was dried and stored at $-80^{\circ} \mathrm{C}$ until analysis.

In case of cell cultures, the quenching metabolism is also fundamental. For Chinese hamster ovary $(\mathrm{CHO})$ cell culture different extraction solvents were tested [8]. Acetonitrile, ethanol, methanol, acetonitrile: water (8:2), ethanol: water (8:2), methanol: water (8:2), $0.1 \mathrm{M}$ formic acid, $0.5 \mathrm{M}$ perchloric acid (PCA) and $0.1 \mathrm{M}$ formic acid in methanol were used. It was found that methanol provided the most efficient extraction (recovery of 53\%). Other interesting result is that extraction solvents containing water caused very broad, split chromatographic peaks. Perchloric acid, neutralised with $\mathrm{KOH}$ was found to be incompatible with LC-MS because of presence of precipitate. Sonication and hexane extraction were also tested; hexane extraction provided greater sample clean-up and better peak shapes; sonication also improved recovery, but as the cooling of plates was unsolved, the sonication was disregarded. Methanol: acetonitrile: water (40:40:20) at $-20{ }^{\circ} \mathrm{C}$ was used for cancer cell lines HCT116 and Calu 6 and methanol: acetonitrile (50:50) at $-20^{\circ} \mathrm{C}$ for cell medium [6]. Sonication was used for cell cultures such as for Hela cells [34] and HCT116 [30]. In an experiment for metabolomic analysis of Plasmodium falciparum lipid related metabolites, two different methods were used depending on ionisation mode in mass spectrometry. After two extraction step by methanol: water (80:20), in the last resuspension, acetonitrile: water $(75: 25)$ was used.

The second step of tissue or cell analysis after arresting metabolism is homogenization. Frozen tissues can be homogenized using a mortar or other physical effect [4, 31, 34]. Sometimes, direct extraction [6] is used where methanol: acetonitrile: water (40:40:20) are the organic solvents, and the extraction is followed by shaking cycle. Extraction solvents are usually the same as for cell cultures and PCA can also be found in some procedures [4] and this needs neutralising steps as well; heated ethanol buffered with $10 \mathrm{mM}$ ammonium acetate can also provide a suitable recovery [31].

Human plasma [6,34], serum $[6,34]$, urine $[6,34]$ and cerebrospinal fluid [34] usually need only a protein precipitation step, which can be performed with methanol:acetonitrile [6] (50:50) or by a simple filtration with a centrifuge filter unit [34].

In a recent publication [12], human acidified blood samples were analysed where collection of blood samples was performed in a sample tube containing $0.5 \mathrm{~N}$ PCA solution. After the sample preparation process $0.5 \mathrm{mM}$ ammonium formate was added to supernatant.

Caruso et al. investigated the preanalytical phase of blood adenine and pyridine nucleotide determination [2]. They performed a double extraction method and two different chromatographic runs for reduced and oxidized forms of nucleotides.

In acid extraction for oxidized form, $\mathrm{K}^{+} /$EDTA treated whole blood was mixed with 7.2\% PCA, and after centrifugation, $1 \mathrm{M}$ borate buffer was used as a neutralizing agent. Borate buffer was compared to carbonate buffer and $\mathrm{KOH}$ as neutralizing agent for acidic extraction, and it was demonstrated that $1 \mathrm{M}$ borate buffer provides a stable $\mathrm{pH} 6.5$ supernatant.

In alkaline extraction for reduced forms, cold, $0.5 \mathrm{M} \mathrm{KOH}$ was added to $\mathrm{K}^{+} /$EDTA treated whole blood. After treatment 


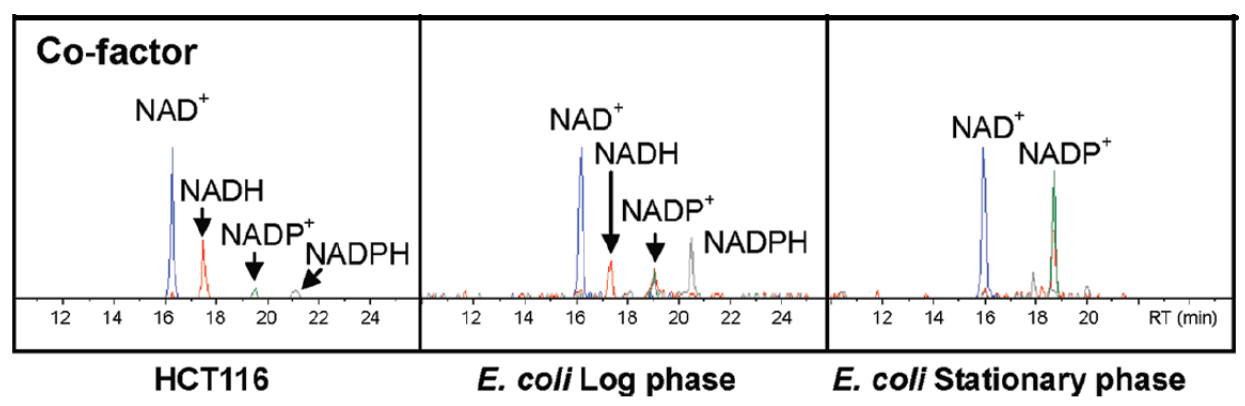

Fig. 3 The MRM chromatograms obtained by using normal phase nanoflow liquid chromatography of pyridine dinucleotides of human HCT116 cells, log phase E.coli and stationary phase E.coli cells [30].

with cold distilled water, ultrafiltration membranes were used to separate the nucleotide containing phase. After ultrafiltration, $1 \mathrm{M} \mathrm{KH}_{2} \mathrm{PO}_{4}$ was used to neutralise the solution. Ultrafiltration time and different ultrafiltration conditions were tested.

\section{Liquid chromatography}

Difficulties in the separation of nucleotides arise from two main sources: the high polarity and the similar chemical structure of these molecules. In developing useful methods, it must be considered that in some cases, molecules only differ in one hydrogen atom. The phosphate moiety also makes the use of reverse phased (RP) LC more difficult.

Anion exchange chromatography (AEX) could be used to separate anionic metabolites, but it has a poor compatibility with MS since in AEX, non-volatile salts are generally used. Salt concentration must be reduced with a membrane suppressor if it is coupled with MS [36].

Normal phase (NP) nanoflow LC was used to separate phosphor-related metabolic changes. Uehara et al. [30] found an amino-propyl silica gel column with basic ammonium carbonate buffer appropriate for the separation of pyridine nucleotides. As ionic and hydrophilic interaction was present between the phosphorus compounds and the solid phase, this method provided good retention and separation (Fig. 3).

However, the use of normal phase is less common in current metabolomics, when LC is coupled to MS. Reversed phase liquid chromatography (RP-HPLC) without ion-pair reagent has not shown good performance either. As mentioned above, nucleotides are highly polar, hence interaction with the stationary phase is insufficient, and metabolites are minimally retained, and poor peak shapes are detected. When separating 160 metabolites of E.coli, Bajad et al. [29] tested two different silica based reversed phase columns, one with embedded polar group and one with ether linked phenyl at three different $\mathrm{pH}$ values, but they found that lack of phosphate in the buffer (phosphate buffer is non-volatile thus incompatible with MS) yielded poor peak shape. However, in case of UV detection which allows use of phosphate buffers, RP-HPLC could be a useful method. An extensive report about preanalytical phase of nucleotide assay applied RP-HPLC with UV detection successfully [2].
Ion-pair (IP) reagents seem to solve the mentioned problems of RP-HPLC, therefore IP-RP-HPLC is one of the most studied method in metabolomics. Use of volatile salts is compatible with MS detection. The principle of ion-pairing is the interaction between the negatively charged nucleotide and the positively charged ion-pair reagent. When using IP reagents, the stationary phase is a usual reversed phase column. Ion-pair reagents are normally alkylamines, such as dibutylammonium acetate (DBAA) [32], tributylamine (TBA) [5-7, 29, 31], dimethylhexylamine (DMHA) [8, 34], dibutylamine (DBA) [4], hexylamine (HA) [7] for negative ionization mode. As nucleotides are detected in negative mode, ion suppression is less problematic. The effect of adding DBAA as an ion-pair reagent is shown in Fig. 4.

Luo et al. [5] investigated the effect of several volatile alkylamine ion-pair reagents, different $\mathrm{pH}$ ranges, and the effect of using methanol and acetonitrile as organic mobile phase. Five different alkylamines, triethylamine (TEA), tripropylamine (TPrA), tributylamine (TBA), tripentylamine (TPeA), and trihexylamine (THA) were tested in the first step at $\mathrm{pH} 6.8$ in the aqueous eluent. Systematic increase of retention was observed with the increase of the length of the alkyl chain. This is caused by the hydrophobic interaction of the alkyl chain with the stationary phase. TEA and TPrA which are alkylamines with short alkyl chain did not offer good resolution, and the use of longer alkyl chains such as TPeA and THA resulted in long retention times and poor peak shapes. After choosing TBA as the best IP reagent, effect of $\mathrm{pH}$ was tested, and $\mathrm{pH} 4.95$ was found to be the optimal $\mathrm{pH}$ to separate 29 metabolites including sugar phosphates, nucleotides and carboxyl acids. It must be mentioned here that changing the $\mathrm{pH}$ between 4.95 and 6.8 did not have effect on the separation of nucleotides due to their low $\mathrm{pK}_{\mathrm{a}}$. Decreasing the $\mathrm{pH}$ was necessary because of their interest in carboxylic acid metabolites. The last step was to optimise the organic eluent. Methanol was chosen as its aspect of being a weaker eluent gave the opportunity of the fine tuning of the mobile phase. Figure 5 shows the combined chromatogram of 29 negatively charged compounds (sugar phosphates, nucleotides, and carboxylic acids), where the peaks of $\operatorname{NAD}(\mathrm{P})(\mathrm{H})$ are highlighted. If the main object is the determination of pyridine dinucleotides, changing the gradient of the elution can shorten the separation. 


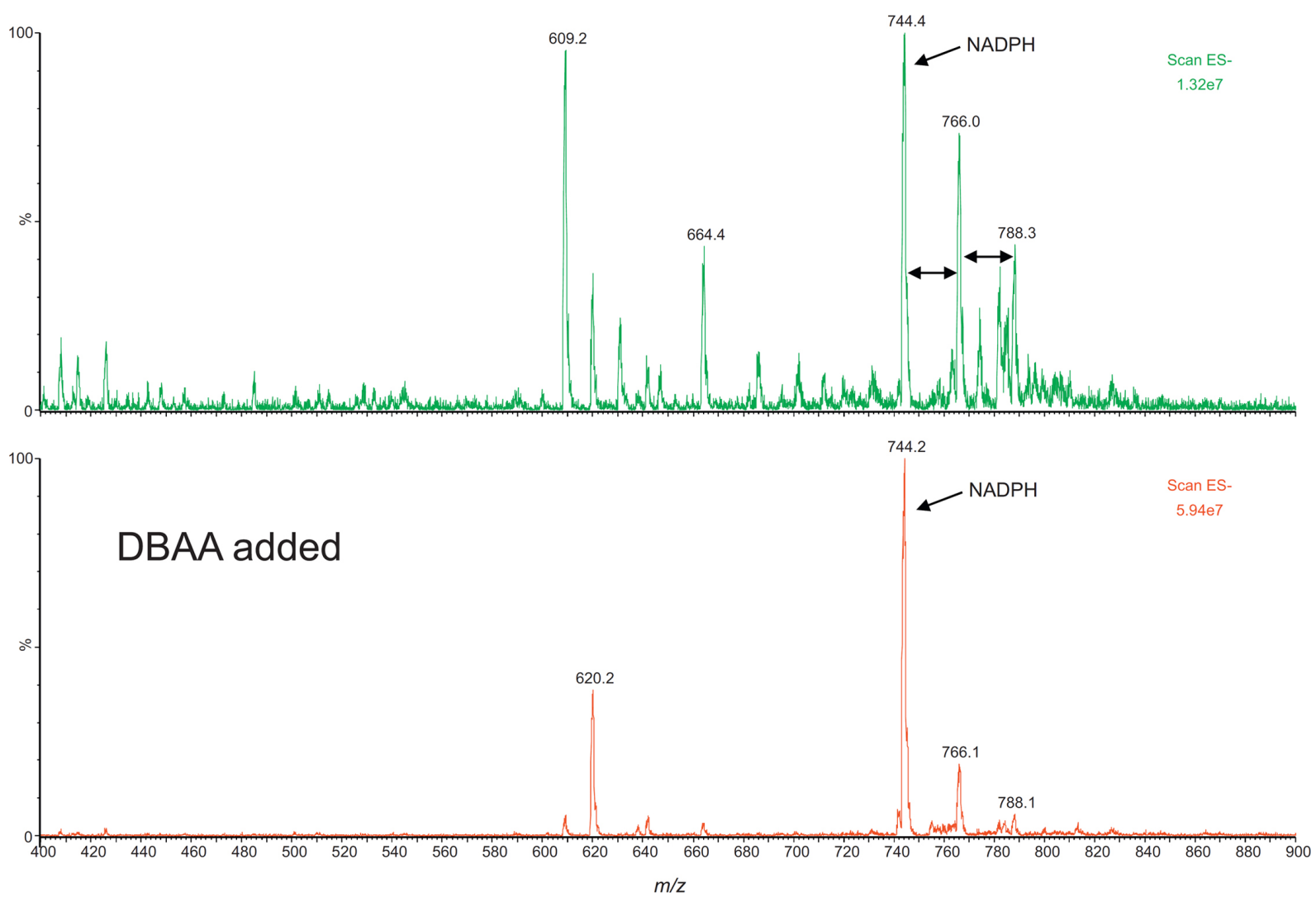

Fig. 4 Effect of DBAA ion-pair reagent on peak height of NADPH [32].

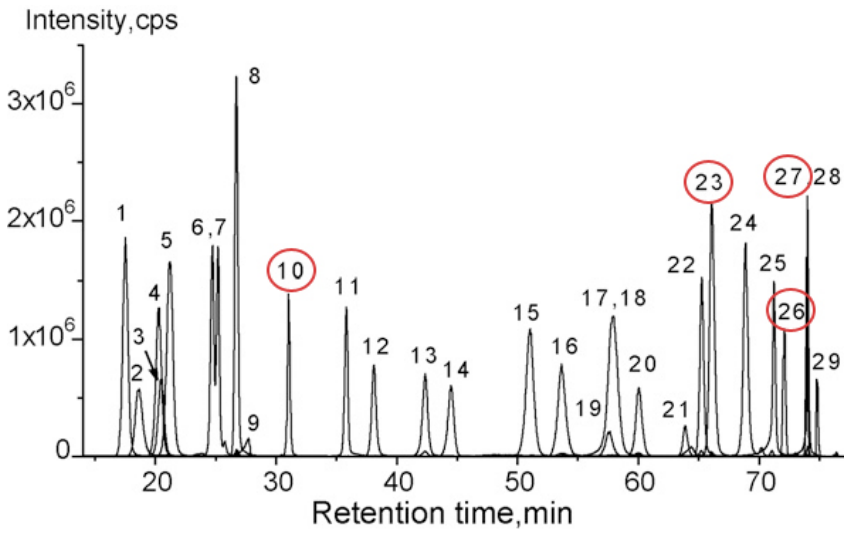

Fig. 5 Combined selective ion chromatogram of 29 metabolites in a standard mixture. The LC-MS/MS method is described in Table 1. Peaks of pyridine dinucleotides: (10) NAD; (23) NADP (26) NADH; (27) NADPH [5].

Optimization of the concentration of IP is fundamental as it can represent a source of contamination of the MS. The concentration can be reduced if the alkyl chain of the used IP is longer as it helps interaction between IP reagent and the apolar stationary phase. The effect of different concentrations of DMHA was tested by Cordell et al. [8]. They varied the concentration of the ion-pair agent between $0.5 \mathrm{mM}$ and $5 \mathrm{mM}$. It was found that the increasing concentration of IP agent caused increasing retention time and better peak shape. Concentration greater than $5 \mathrm{mM}$ did not improve the separation. Effect of mobile phase $\mathrm{pH}$ was also tested. It was noticed that increasing $\mathrm{pH}$ increased retention time and the $\mathrm{pH}$ had influence on peak shapes as well. At $\mathrm{pH}$ below 3, compounds eluted near dead time, and peak broadening was observed. Figure 6 represents the simultaneous separation of 24 nucleotides for standard solution (a), and for biological sample (b). The chromatogram shows the most important advantage of MS detection: the peaks which overlap in the combined chromatograms, in fact do not overlap.

To avoid contamination, Seifar et al. [32] diverted the effluent of the analytical column to waste at the beginning and in the end of the separation, which prevented the ESI source and the MS from being contaminated.

The other most important chromatographic system, which is used in metabolic profiling, is hydrophilic interaction chromatography (HILIC). In HILIC, the retention mechanism is partitioning, and water is used as the strong eluent [37]. There can be found three main types of HILIC columns, the neutral (no electrostatic interaction), charged (strong electrostatic interaction) and zwitterionic (weak electrostatic interaction). A very successful application of HILIC was reported by Bajad et al. [29]. Three different HILIC columns were compared, cyano column at acidic $\mathrm{pH}$, silica column and amino column at $\mathrm{pH} 9$. They found that the cyano and silica column had minimal retention and poor peak shape for multi-phosphorylated compounds, but an amino-modified HILIC column at $\mathrm{pH} 9$ gave detectable peaks for triphosphates as well with a good peak shape. After optimization, 69 metabolites from E. coli were quantified. In the metabolomic analysis of Plasmodium falciparum, which 
a)

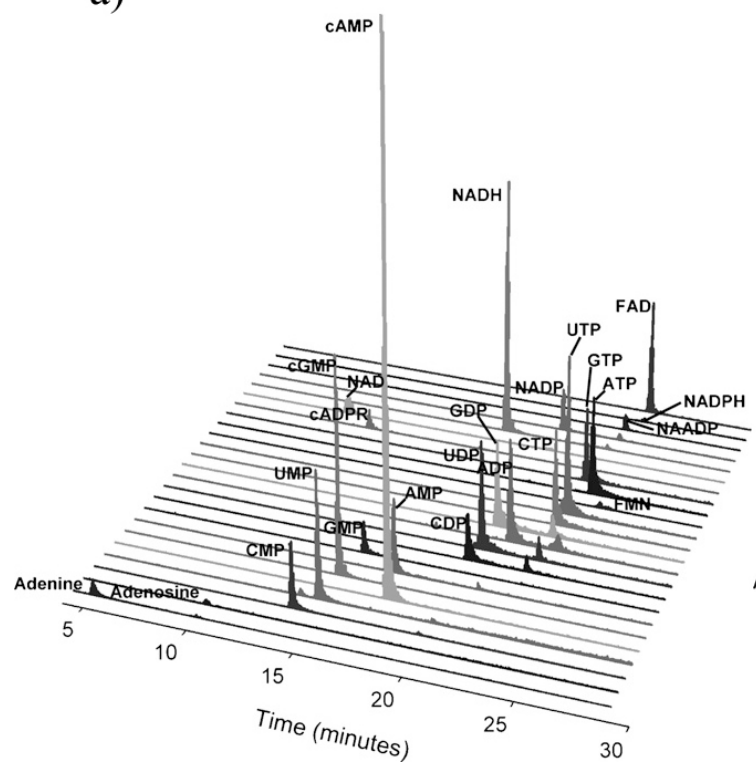

b)

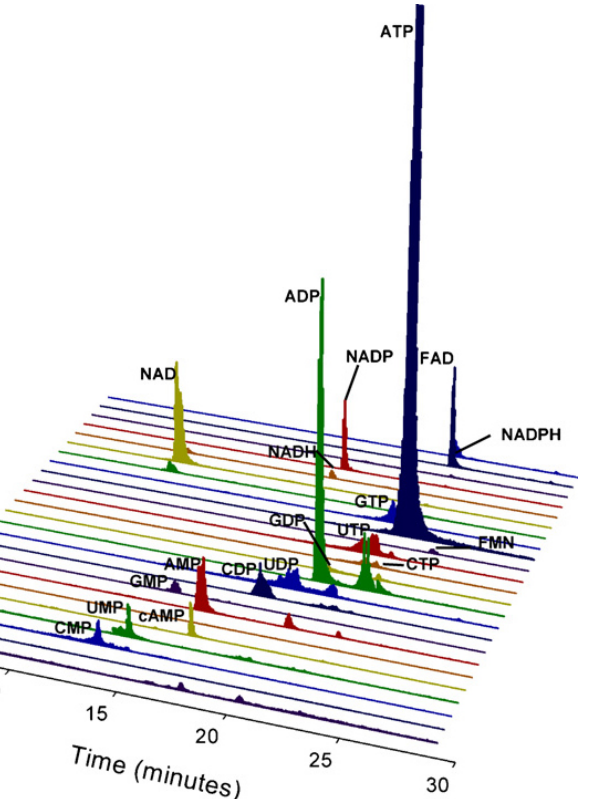

Fig. 6 Results of MRM scan (dwell 0.2 s) of simultaneous detection of 24 nucleotides in standard solution

(a) and for biological sample in control conditions (b) [8]. LC-MS method is described in Table 1.

is the causative agent of malaria, a Luna NH2 HILIC column under alkaline conditions in negative ionization mode was used [35]. Their method was complementary with ion-pair chromatography, and a total of 35 compounds were quantified. Many reports were published about comparison between IP-RP-HPLC and HILIC-HPLC. Michopoulos et al. [6] found that use of ionpair agents provided better separation and peak shapes than HILIC. Peak tailing was observed for multi-phosphorylated compounds, which was increasing with the degree of phosphorylation. In spite of these facts, good resolution was observed for reduced and oxidized forms of $\mathrm{NAD}^{+}$and $\mathrm{NADP}^{+}$.

Myint et al. [34] reported a practical method for polar anionic metabolites using nano-LC/MS system. A polyaminebonded polymer-based apHera NH2 column was applied, and during optimization, it was observed that traces of metal caused a binding to the column for highly polar compounds (citric acid or ATP). A chelating agent, EDTA was added in 85 pmol concentration to remove metallic impurities which may come from the stainless steel of HPLC hardware. By using EDTA, no further peak tailing was observed and the previously disappearing peaks were detected in every analysis. A titania column was used to separate nucleotides and their pathway intermediates [21]. Titania's mechanical and $\mathrm{pH}$ stability is better than of silica. It acts as Lewis acid and this way it has a strong affinity for phosphonate groups. This property makes it a very good possibility in separating nucleotides. In their report, Zhou et al. examined the effects of mobile phase composition $(\%(\mathrm{v} / \mathrm{v}))$ of $\mathrm{ACN}$ in sample, eluent $\mathrm{pH}$ and eluent anion. By optimizing the titania column, fifteen nucleotides and their intermediates were separated.

IP-RP-HPLC and HILIC can act as complementary methods in profiling metabolites. The need for high proportions of organic solvents in separations by HILIC can confer benefit as it intensifies MS signal by facilitating ionization, but it can be a disadvantage as well. In many cases, nucleotides are present in a very low concentration in biological samples, and diluting them to the same solvent composition as the eluent can lower the concentration below the working concentration range.

Recently, beside the common stationary phases, many other possibilities are gaining popularity. One of the most promising phases is graphitic carbon column. The retention mechanism is very complex, pi-pi interactions and dispersive interactions between the stationary phase and the aromatic solutes seem to be the main components [38]. PGC is still not so much used, but there can be found reports about its possible applications. When comparing HILIC and IP-RP-HPLC, Michopoulos et al. [6] examined PGC columns as well, and they found it applicable for pyridine nucleotides. When $50 \mathrm{mM}$ ammonium hydrogen carbonate was added to the eluent containing $60 \% \mathrm{ACN}$ and $40 \%$ water, improvement was observed in elution of $\mathrm{NAD}^{+}, \mathrm{NADH}$, $\mathrm{NADP}^{+}, \mathrm{NADPH}$ and AMP. Despite the promising results in separation of pyridine nucleotides, the separation method was not examined in further studies since they were focusing on a greater number of metabolites to be determined.

Another approach was presented with respect to quantitative metabolomics in Methylobacterium extorquens [33]. Comparison was performed between a pentafluorophenyl-propyl column and a Luna NH2 HILIC column. Reversed phase mode provided a suitable separation of all tested analytes. After examining the effect of buffers, they proved that ammonium formate and ammonium acetate in acidic $\mathrm{pH}$ lowered retention and the separation deteriorated. The use of $0.1 \%$ aqueous formic acid/acetonitrile gradient provided the best results with good retention, and good peak shapes. Although $\mathrm{NADP}^{+}$and 
ATP showed 4 times lower sensitivity, compared to NADH and NADPH, 5-10 times higher sensitivities were observed than with the use of the HILIC column.

\section{Mass spectrometry}

Detection in complex biological matrices, such as blood, urine and tissues, is complicated by the presence of numerous contaminants, which makes the precise determination of selected components more difficult or even impossible without a highly sensitive and selective method. This is where the disadvantages of UV detection are the most evident. MS can differentiate molecules based upon their mass, which allows the use of isotope labelled internal standards. The biggest drawback of MS is the inability to distinguish isobaric components, such as sugar phosphates or cis/trans molecules. Despite this drawback, coupling LC to MS can provide a highly sensitive and widely usable method in qualification and quantification of metabolome. Coupling the two instruments was the biggest challenge due to the high flow rate and to the possibility of contaminating the MS. Electrospray ionization (ESI) has become the most important ionization technique in bioanalytical studies. For detection of nucleotides, usually negative ESI is applied since these molecules have acidic groups, but reports describing positive mode also can be found [12].

In untargeted metabolomics, very high resolution is essential, thus use of FTIR MS is established, but in targeted profiling, a triple quadrupole (QQQ) may be the best choice. QQQ has a lower resolution, but can reduce the background noise hence its sensitivity is outstanding. Triple quadrupole is usually used in selected reaction monitoring (SRM) $[5,29,35]$ or in multiple reaction monitoring (MRM) [6-9, 12, 30-34] Bajad et al. [29] profiled the metabolome of E.coli, and since many of the metabolites can be ionized in positive mode, the 50-minute LC runs were divided into nine time segments, five of which were in positive mode and four segments were in negative mode. Compounds eluting at the boundaries had SRM scan in both time segments.

Modern softwares provide the possibility of automatic optimization, which can facilitate the selection of the best daughter ion(s), but in some cases, manual optimization is recommended, especially in case of compounds with very similar structure - this is the case of nucleotides. NAD ${ }^{+}$and NADH differ by only one hydrogen, and the only difference between $\mathrm{NAD}^{+}$and $\mathrm{NADP}^{+}$is a phosphate group. When Cordell et al. [8] developed a method for determination of twenty nucleotides, efficiency of both ESI+ and ESI- were examined. Because DMHA was used as ion-pair agent, the possibility of detecting adduct ions of nucleotides and DMHA allowed the testing of ESI+. However, protonated DMHA caused a high background interference in the ESI+ spectra. [M+DMHA]+ was found to be the most abundant relevant ion, but since in ESI- mode the background was much lower, signal/noise ratio was much higher in
ESI-. After determining dominant product ions of each nucleotide, different ionization parameters were optimized.

Heated and unheated electrospray ionization were compared by Lu et al. [7]. While in the unheated ESI source, the auxiliary gas and sheath gas is at ambient temperature, in heated ESI sources (HESI) temperature is usually between 200 $600{ }^{\circ} \mathrm{C}$. As high temperature accelerates the evaporation of sample solution emitted from the metal needle, it increases the ionization efficiency. During their experiment, Lu et al. investigated the effect of HESI for targeted metabolomics. E. coli cellular extract was studied, and two different mass spectrometers (Thermo Scientific TSQ Quantum Ultra instrument with unheated or heated ESI sources, and an AppliedBiosystems API 4000 instrument with Turbo VTM source). The experiment was carried out in positive and in negative mode as well. They found that with the use of heated ESI, the sensitivity was at least five times greater than with unheated ESI. Although noise increased, the signal-to-noise ratio was enhanced significantly. It must be noted that the effect of HESI depends on the compound: for methionine, a 25-fold increase in absolute ion counts was observed, but for NAD, HESI did not provide improvement as seen in Fig. 7. Heat degradation of metabolites also must be taken into accounts, but this is a subject of further experiments.
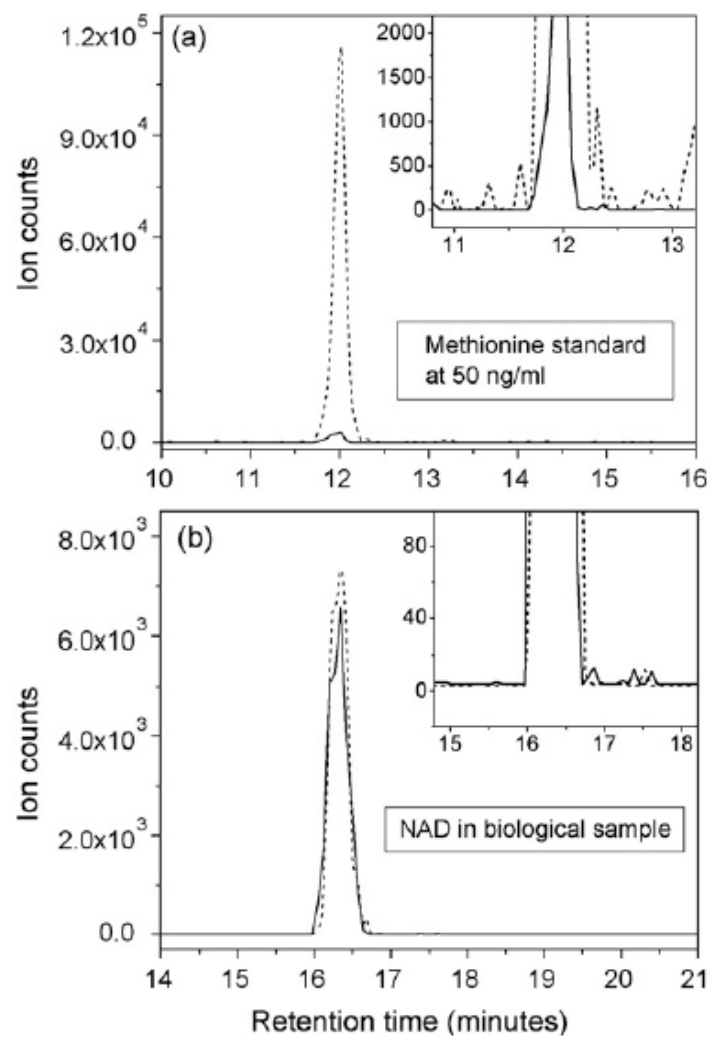

Fig. 7 Comparison of heated and non-heated ESI. As reported, for methionine standard (a), a huge increase in intensity was observed, while for NAD ${ }^{+}$ in biological sample (b) the increase was insignificant. However, as this is compound-dependent, it is worth to check the effect of heating [7]. 


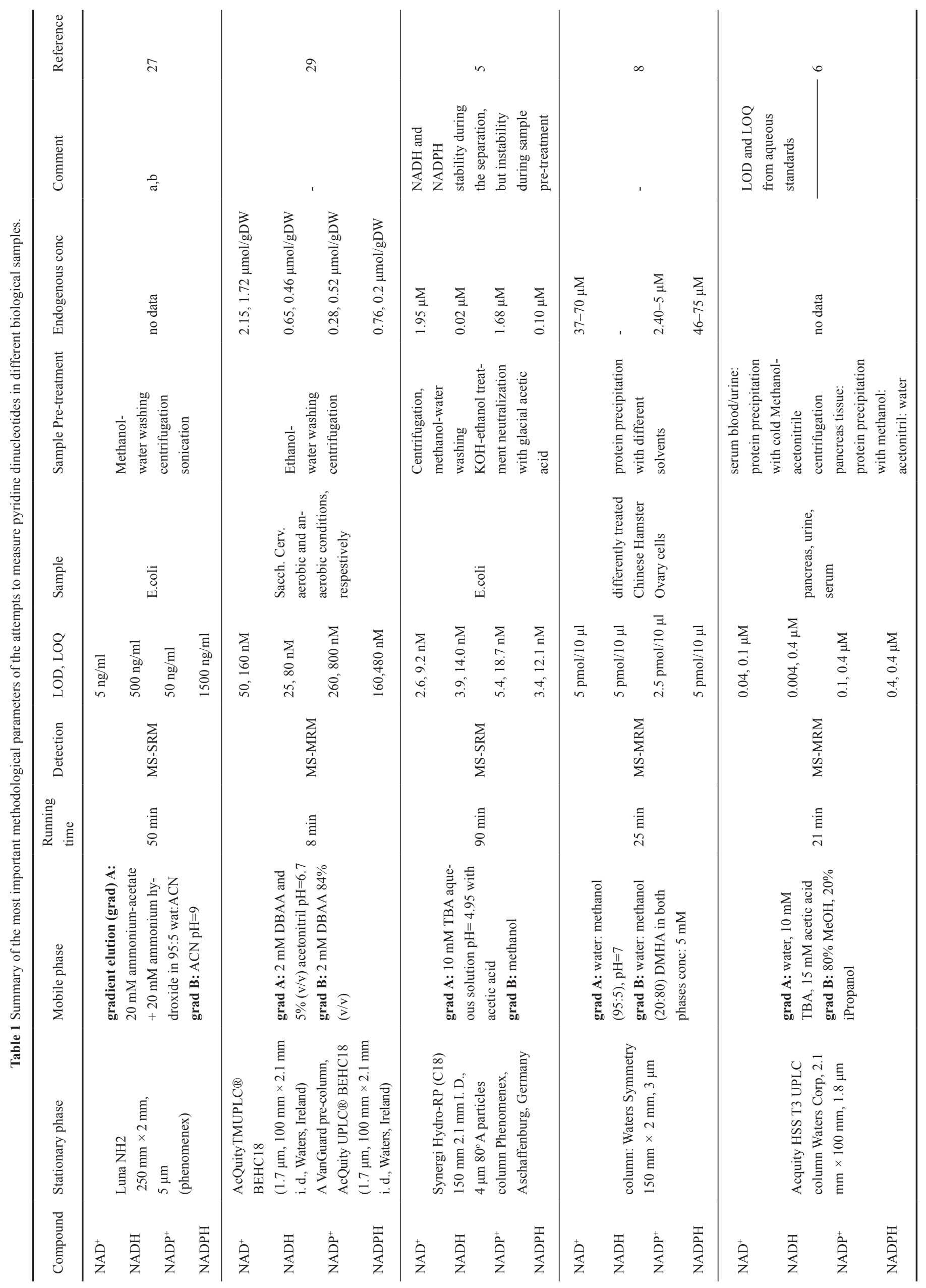




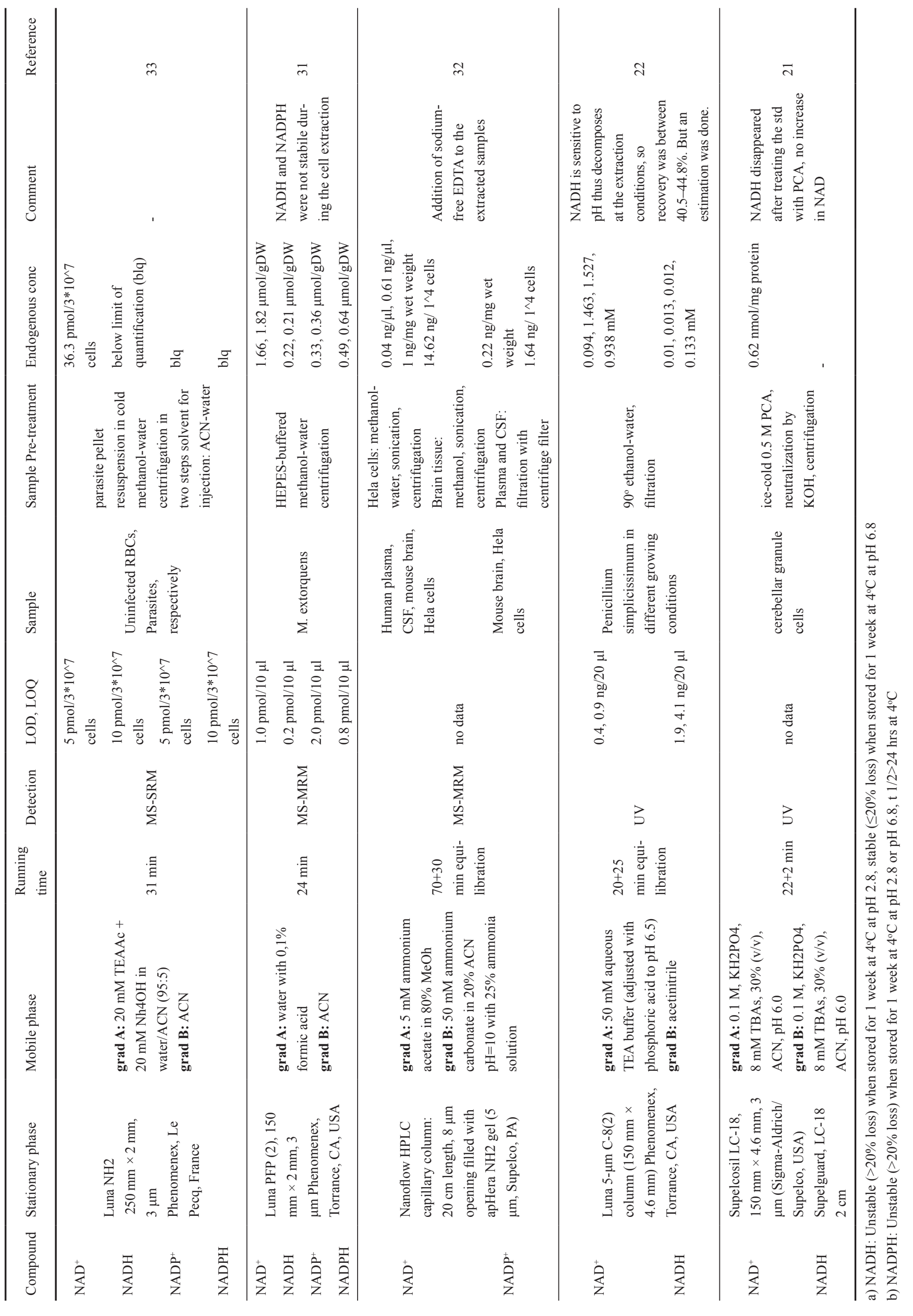




\section{Calibration curves and endogenous concentration}

Stock solutions are usually prepared in pure water $[4,5,22$, $24,31,35]$ or in water: methanol 50:50 [30]. After the preparation of a $10 \mathrm{mM}$ standard solution, Klawitter et al. [4] stored it at $-80^{\circ} \mathrm{C}$, and prepared working solutions every day which had to be used within two hours at room temperature.

Calibration concentration during the measurements vary among the experiments. In some cases, $1.5 \mathrm{nM}$ was the lower limit of calibration [5, 29], but usually the calibration curves lie between $0.1 \mu \mathrm{M}$ and $10 \mu \mathrm{M}$. Top concentration of calibration was $200 \mu \mathrm{M}$ in one case [9]. Linearity is usually tested with standard solutions, or treating standard solutions with the sample preparation protocol [2]. Endogenous concentration is generally determined by spiking the sample with a mix of the compounds at different concentrations, or by using isotope labelled internal standards, which are obtained from isotope labelled cell extract $[9,32,33]$.

Despite the significant variability in the endogenous pyridine dinucleotide levels, the concentrations of these cofactors are always in a very low range in the biological samples. Hence, having a good lowest limit of detection (LOD) and lowest limit of quantification (LOQ) is essential. LOD is usually defined as the concentration where the signal/noise ratio is 3 , and at LOQ concentration, the ratio is 10 . The results obtained by several research groups using different samples can be seen in Table 1 . It may be noticed, that LOD, LOQ and endogenous concentrations are given in different units; the choice usually depends on the type of the sample (tissue, cell culture, etc.), or - especially for LOD and LOQ - whether it is given in concentration or in amount of substance on column.

\section{Stability of pyridine dinucleotides}

Instability of pyridine dinucleotides is one of the biggest challenges to be met when studying these molecules. Stability of nicotinamide cofactors in solution was tested by Wong et al. [39]. It was shown that reduced forms are more stable in alkaline solution, and oxidized forms are more stable in acidic solutions. This is caused by an acid-catalysed hydration of $\mathrm{NADH}$. Other strong nucleophiles, such as $\mathrm{SO}_{3}{ }^{2-}$ or $\mathrm{CN}^{-}$also induce decomposition of the oxidized forms. At low $\mathrm{pH}$, where the concentration of sodium-acetate buffer is $0.1 \mathrm{M}$, the decomposition of NADPH is three times faster than of NADH. In some cases, disappearance of the reduced forms was observed during sample preparation $[23,33]$. Treating the standard solution of NADH with perchloric acid, it disappeared, but increase of $\mathrm{NAD}^{+}$level was not observed [23]. Yang et al. applied a sample preparation method that turned out to be too drastic for the measurement of the level of reduced forms [33]. As NADPH was unstable at $\mathrm{pH} 2.8$ and 6.8, Bajad et al. decided to leave it out from further analyses. Furthermore, poor performance was observed for NADH as well. When examining E.coli, the applied LC-MS/MS method provided good separation for all of the four nucleotides in standard solution due to the mild conditions. Despite this, the observed concentration of NADH and NADPH in E.coli samples was very low. The conditions during the sample preparation (the use of $\mathrm{KOH}$ and glacial acetic acid) might have caused the degradation of the reduced forms [5].

\section{Application and conclusion}

Pyridine dinucleotides play an essential role in metabolism, and their ratio is fundamental in normal functioning of cells or cell compartments. By monitoring levels of these nucleotides under varying conditions, more information could be achieved about the pathomechanism of metabolic disorders, aging process, and effects of drug candidates, hence it could help develop treatment for a variety of diseases.

Concentration of $\mathrm{NAD}^{+}, \mathrm{NADH}, \mathrm{NADP}^{+}$and $\mathrm{NADPH}$ indicates the current status of cells hence it can be a marker of numerous disorders. Determination of adenine dinucleotide levels under different nutritional conditions clearly revealed the effect of starvation or a high-sucrose diet [10]. Thus, accurate measurement of pyridine dinucleotide redox couples would largely facilitate researches about metabolic disorders and it could be applied in clinical diagnosis as well.

In the last decade, a wide variety of studies have been conducted in order to profile metabolic status of different bacteria, yeast, mammalian cell cultures and tissues. A great number of techniques were used, and it can be declared that HPLC-MS has become the leading method. Several studies were performed in order to substitute RP-HPLC, or HILIC, such as pentafluorophenylpropyl column, titania column, or graphitic carbon columns. Capillary electrophoresis may be a potential separation technique, as pyridine dinucleotides are negatively charged molecules. Nano-LC-MS can also represent a potential future technique. Despite the numerous reports, there are still challenges to face. Several studies focused only on oxidized forms of nucleotides, and among those who decided to determine the concentration of both oxidized and reduced forms, many groups reported poor performance or were unable to quantify the concentration of reduced forms due to instability of these compounds $[6,9,22,29,32,33]$. Due to the different characteristics of reduced and oxidized forms, simultaneous determination of both forms is problematic, hence it may be possible that biological matrices need to be prepared in two different ways for separate analysis of $\mathrm{NAD}(\mathrm{P})^{+}$and $\mathrm{NAD}(\mathrm{P})$ $\mathrm{H}$. Therefore, the sample preparation protocols suitable for HPLC-MS quantification of all the four relevant pyridine dinucleotides still remain to be elaborated.

\section{Acknowledgement}

The authors thank the Hungarian Scientific Research Fund (OTKA 106060) for its support. 


\section{References}

[1] Sun, F. F., Dai, C. Y., Xie, J. S., Hu, X. "Biochemical Issues in Estimation of Cytosolic Free NAD/NADH Ratio." PLoS One. 7(5), e34525. 2012. DOI: 10.1371/journal.pone.0034525

[2] Caruso, R., Campolo, J., Dellanoce, C., Mariele, R., Parodi, O., Accinni, R. "Critical study of preanalytical and analytical phases of adenine and pyridine nucleotide assay in human whole blood." Analytical Biochemistry. 330(1), pp. 43-51. 2004. DOI: 10.1016/j.ab.2004.03.063

[3] Kereszturi, E., Kalman, F. S., Kardon, T., Csala, M., Banhegyi, G. "Decreased prereceptorial glucocorticoid activating capacity in starvation due to an oxidative shift of pyridine nucleotides in the endoplasmic reticulum." FEBS Letters. 584(22), pp. 4703-4708. 2010.

DOI: $10.1016 /$ j.febslet.2010.10.053

[4] Klawitter, J., Schmitz, V., Klawitter, J., Leibfritz, D., Christians, U. "Development and validation of an assay for the quantification of 11 nucleotides using LC/LC-electro spray ionization-MS." Analytical Biochemistry. 365(2), pp. 230-239. 2007. DOI: 10.1016/j.ab.2007.03.018

[5] Luo, B., Groenke, K., Takors, R., Wandrey, C., Oldiges, M. "Simultaneous determination of multiple intracellular metabolites in glycolysis, pentose phosphate pathway and tricarboxylic acid cycle by liquid chromatography-mass spectrometry." Journal of Chromatography A. 1147(2), pp. 153-164. 2007. DOI: 10.1016/j.chroma.2007.02.034

[6] Michopoulos, F., Whalley, N., Theodoridis, G., Wilson, I. D., Dunkley, T. P. J., Critchlow, S. E. "Targeted profiling of polar intracellular metabolites using ion-pair-high performance liquid chromatography and -ultra high performance liquid chromatography coupled to tandem mass spectrometry: Applications to serum, urine and tissue extracts." Journal of Chromatography A. 1349, pp. 60-68. 2014.

DOI: 10.1016/j.chroma.2014.05.019

[7] Lu, W., Bennett, B. D., Rabinowitz, J. D. "Analytical strategies for LC-MS-based targeted metabolomics." Journal of Chromatography B Analytical Technologies in the Biomedical and Life Sciences. 871(2), pp. 236-242. 2008. DOI: 10.1016/j.jchromb.2008.04.031

[8] Cordell, R. L., Hill, S. J., Ortori, C. A., Barrett, D. A. "Quantitative profiling of nucleotides and related phosphate-containing metabolites in cultured mammalian cells by liquid chromatography tandem electrospray mass spectrometry." Journal of Chromatography B - Analytical Technologies in the Biomedical and Life Sciences. 871(1), pp. 115-124. 2008. DOI: $10.1016 /$ j.jchromb.2008.07.005

[9] Trammell, S. A., Brenner, C. "Targeted, LCMS-based Metabolomics for Quantitative Measurement of NAD(+) Metabolites." Computational and Structural Biotechnology Journal. 4, e201301012. 2013. DOI: $10.5936 / \mathrm{csbj} .201301012$

[10] Veech, R. L., Eggleston, L. V., Krebs, H. A. "The redox state of free nicotinamide-adenine dinucleotide phosphate in the cytoplasm of rat liver." Biochemical Journal. 115(4), pp. 609-619. 1969.

[11] Gomes, A. P., Price, N. L., Ling, A. J. Y., Moslehi, J. J., Montgomery, M. K., Rajman, L., White, J. P., Teodor, J. S., Wrann, C. D., Hubbard, B. P., Mercken, E. M., Palmeira, C. M., de Cabo, R., Rolo, A. P., Turner, N., Bell, E. L., Sinclair, D. A. "Declining NAD(+) Induces a Pseudohypoxic State Disrupting Nuclear-Mitochondrial Communication during Aging." Cell. 155(7), pp. 1624-1638. 2013. DOI: 10.1016/j.cell.2013.11.037

[12] Liu, L. L., Cui, Z. Y., Deng, Y. Z., Dean, B., Hop, C. E. C. A., Liang, X. R. "Surrogate analyte approach for quantitation of endogenous $\mathrm{NAD}(+)$ in human acidified blood samples using liquid chromatography coupled with electrospray ionization tandem mass spectrometry." Journal of Chromatography B-Analytical Technologies in the Biomedical and Life Sciences. 1011, pp. 69-76. 2016. DOI: 10.1016/j.jchromb.2015.12.040
[13] Csala, M., Banhegyi, G., Benedetti, A. "Endoplasmic reticulum: A metabolic compartment." FEBS Letters. 580(9), pp. 2160-2165. 2006. DOI: 10.1016/j.febslet.2006.03.050

[14] Banhegyi, G., Benedetti, A., Csala, M., Mandl, J. "Stress on redox." FEBS Letters. 581(19), pp. 3634-3640. 2007. DOI: $10.1016 /$ j.febslet.2007.04.028

[15] Zambo, V., Simon-Szabo, L., Szelenyi, P., Kereszturi, E., Banhegyi, G., Csala, M. "Lipotoxicity in the liver." World Journal of Hepatology. 5(10), pp. 550-557. 2013. DOI: 10.4254/wjh.v5.i10.550

[16] Senesi, S., Legeza, B., Balazs, Z., Csala, M., Marcolongo, P., Kereszturi, E., Szelenyi, P., Egger, C., Fulceri, R., Mandl, J., Giunti, R., Odermatt, A., Banhegyi, G., Benedetti, A. "Contribution of Fructose-6-Phosphate to Glucocorticoid Activation in the Endoplasmic Reticulum: Possible Implication in the Metabolic Syndrome." Endocrinology. 151(10), pp. 4830-4839. 2010. DOI: 10.1210/en.2010-0614

[17] Margittai, E., Enyedi, B., Csala, M., Geiszt, M., Banhegyi, G. "Composition of the redox environment of the endoplasmic reticulum and sources of hydrogen peroxide." Free Radical Biology \& Medicine. 83, pp. 331-340. 2015. DOI: 10.1016/j.freeradbiomed.2015.01.032

[18] Piccirella, S., Czegle, I., Lizak, B., Margittai, E., Senesi, S., Papp, E., Csala, M., Fulceri, R., Csermely, P., Mandl, J., Benedetti, A., Banhegyi, G. "Uncoupled redox systems in the lumen of the endoplasmic reticulum - Pyridine nucleotides stay reduced in an oxidative environment." Journal of Biological Chemistry. 281(8), pp. 4671-4677. 2006.

[19] Bublitz, C., Lawler, C. A. "The Levels of Nicotinamide Nucleotides in Liver-Microsomes and Their Possible Significance to the Function of Hexose Phosphate Dehydrogenase." Biochemical Journal. 245(1), pp. 263-267. 1987.

[20] Odermatt, A., Atanasov, A. G., Balazs, Z., Schweizer, R. A. S., Nashev, L. G., Schuster, D., Langer, T. "Why is 11 beta-hydroxy steroid dehydrogenase type 1 facing the endoplasmic reticulum lumen? Physiological relevance of the membrane topology of 11 beta-HSD1." Molecular and Cellular Endocrinology. 248(1-2), pp. 15-23. 2006. DOI: 10.1016/j.mce.2005.11.040

[21] Zhou, T., Lucy, C. A. "Hydrophilic interaction chromatography of nucleotides and their pathway intermediates on titania." Journal of Chromatography A. 1187(1-2), pp. 87-93. 2008.

DOI: $10.1016 /$ j.chroma.2008.02.027

[22] Cichna, M., Daxecker, H., Raab, M. "Determination of 18 nucleobases, nucleosides and nucleotides in human peripheral blood mononuclear cells by isocratic solvent-generated ion-pair chromatography." Analytica Chimica Acta. 481(2), pp. 245-253. 2003.

DOI: $10.1016 / \mathrm{S} 0003-2670(03) 00081-3$

[23] Giannattasio, S., Gagliardi, S., Samaja, M., Marra, E. "Simultaneous determination of purine nucleotides, their metabolites and beta-nicotinamide adenine dinucleotide in cerebellar granule cells by ion-pair high performance liquid chromatography." Brain Research Protocols. 10(3), pp. 168-174. 2003. DOI: 10.1016/S1385-299x(02)00215-5

[24] Ganzera, M., Vrabl, P., Worle, E., Burgstaller, W., Stuppner, H. "Determination of adenine and pyridine nucleotides in glucose-limited chemostat cultures of Penicillium simplicissimum by one-step ethanol extraction and ion-pairing liquid chromatography." Analytical Biochemistry. 359(1), pp. 132-140. 2006. DOI: 10.1016/j.ab.2006.09.012

[25] Markuszewski, M. J., Britz-McKibbin, P., Terabe, S., Matsuda, K., Nishioka, T. "Determination of pyridine and adenine nucleotide metabolites in Bacillus subtilis cell extract by sweeping borate complexation capillary electrophoresis." Journal of Chromatography A. 989(2), pp. 293-301. 2003. 
[26] Soga, T., Ueno, Y., Naraoka, H., Ohashi, Y., Tomita, M., Nishioka, T. "Simultaneous determination of anionic intermediates for Bacillus subtilis metabolic pathways by capillary electrophoresis electrospray ionization mass spectrometry." Analytical Chemistry. 74(10), pp. 2233-2239. 2002.

[27] Palfi, M., Halasz, A. S., Tabi, T., Magyar, K., Szoko, E. "Application of the measurement of oxidized pyridine dinucleotides with high-performance liquid chromatography-fluorescence detection to assay the uncoupled oxidation of NADPH by neuronal nitric oxide synthase." Analytical Biochemistry. 326(1), pp. 69-77. 2004. DOI: 10.1016/j.ab.2003.11.010

[28] Becker, S., Kortz, L., Helmschrodt, C., Thiery, J., Ceglarek, U. "LC-MS-based metabolomics in the clinical laboratory." Journal of Chromatography B - Analytical Technologies in the Biomedical and Life Sciences. 883-884, pp. 68-75.2012. DOI: 10.1016/j.jchromb.2011.10.018

[29] Bajad, S. U., Lu, W., Kimball, E. H., Yuan, J., Peterson, C., Rabinowitz, J. D. "Separation and quantitation of water soluble cellular metabolites by hydrophilic interaction chromatography-tandem mass spectrometry." Journal of Chromatography A. 1125(1), pp. 76-88. 2006.

DOI: 10.1016/j.chroma.2006.05.019

[30] Uehara, T., Yokoi, A., Aoshima, K., Tanaka, S., Kadowaki, T., Tanaka, M., Oda, Y. "Quantitative phosphorus metabolomics using nanoflow liquid chromatography-tandem mass spectrometry and culture-derived comprehensive global internal standards." Analytical Chemistry. 81(10), pp. 3836-3842. 2009. DOI: 10.1021/ac9002062

[31] Buescher, J. M., Moco, S., Sauer, U., Zamboni, N. "Ultrahigh performance liquid chromatography-tandem mass spectrometry method for fast and robust quantification of anionic and aromatic metabolites." Analytical Chemistry. 82(11), pp. 4403-4412. 2010. DOI: 10.1021/ac100101d

[32] Seifar, R. M., Ras, C., Deshmukh, A. T., Bekers, K. M., Suarez-Mendez, C. A., da Cruz, A. L., van Gulik, W. M., Heijnen, J. J. "Quantitative analysis of intracellular coenzymes in Saccharomyces cerevisiae using ion pair reversed phase ultra high performance liquid chromatography tandem mass spectrometry." Journal of Chromatography A. 1311, pp. 115-120. 2013. DOI: 10.1016/j.chroma.2013.08.076
[33] Yang, S., Sadilek, M., Lidstrom, M. E. "Streamlined pentafluorophenylpropyl column liquid chromatography-tandem quadrupole mass spectrometry and global (13)C-labeled internal standards improve performance for quantitative metabolomics in bacteria." Journal of Chromatography A. 1217(47), pp. 7401-7410. 2010.

DOI: $10.1016 /$ j.chroma.2010.09.055

[34] Myint, K. T., Uehara, T., Aoshima, K., Oda, Y. "Polar anionic metabolome analysis by nano-LC/MS with a metal chelating agent." Analytical Chemistry. 81(18), pp. 7766-7772. 2009. DOI: 10.1021/ac901269h

[35] Duy, S. V., Besteiro, S., Berry, L., Perigaud, C., Bressolle, F., Vial, H. J., Lefebvre-Tournier, I. "A quantitative liquid chromatography tandem mass spectrometry method for metabolomic analysis of Plasmodium falciparum lipid related metabolites." Analytica Chimica Acta. 739, pp. 47-55. 2012. DOI: 10.1016/j.aca.2012.06.016

[36] Seifar, R. M., Ras, C., van Dam, J. C., van Gulik, W. M., Heijnen, J. J., van Winden, W. A. "Simultaneous quantification of free nucleotides in complex biological samples using ion pair reversed phase liquid chromatography isotope dilution tandem mass spectrometry." Analytical Biochemistry. 388(2), pp. 213-219. 2009. DOI: 10.1016/j.ab.2009.02.025

[37] Alpert, A. J. "Hydrophilic-Interaction Chromatography for the Separation of Peptides, Nucleic-Acids and Other Polar Compounds." Journal of Chromatography. 499, pp. 177-196. 1990. DOI: 10.1016/S0021-9673(00)96972-3

[38] Hanai, T. "Separation of polar compounds using carbon columns." Journal of Chromatography A. 989(2), pp. 183-196. 2003. DOI: 10.1016/S0021-9673(02)02017-4

[39] Wong, C. H., Whitesides, G. M. "Enzyme-Catalyzed Organic-Synthesis $\mathrm{Nad}(\mathrm{P}) \mathrm{H}$ Cofactor Regeneration by Using Glucose-6-Phosphate and the Glucose-6-Phosphate-Dehydrogenase from Leuconostoc-Mesenteroides." Journal of the American Chemical Society. 103(16), pp. 4890-4899. 1981. DOI: $10.1021 / \mathrm{Ja} 00406 \mathrm{a} 037$ 